\title{
Factorisation in diffraction
}

\author{
Alice Valkárová ${ }^{a *}$, on behalf of the H1 and ZEUS Collaborations. \\ ${ }^{a}$ Institute of Particle and Nuclear Physics of the Faculty of Mathematics and Physics of Charles \\ University, V Holešovičkách 2, 18000 Praha 8, Czech Republic
}

Recent experimental data on dijet cross sections in diffractive photoproduction at HERA collider were analysed with emphasis on QCD factorisation breaking effects. Dijet photoproduction data of jets with low transverse energy $E_{\mathrm{T} 1}>5 \mathrm{GeV}$ indicated a possible dependence on $E_{\mathrm{T}}$ of the suppression of jet production with respect to NLO QCD predictions. However, this suppression exhibited no significant dependence on the fraction $x_{\gamma}$ of the photon four-momentum entering the hard process. The ratio of diffractive to inclusive cross section for dijets in photoproduction was also measured for the first time.

\section{Factorisation and diffractive parton dis- tribution functions}

The first observation of deep inelastic scattering (DIS) at HERA containing a large gap in the pseudorapidity distributions of final hadrons [1] has renewed the interest in understanding of diffraction. HERA has made it possible to study diffraction not only in a wide range of exchanged photon virtualities $Q^{2}$ from the region of photoproduction, $Q^{2} \sim 0$, to the DIS region with $Q^{2} \gg 0$, but also for many processes like jet- or vector meson-production. In such diffractive interactions the proton stays intact or dissociates into a low mass state (Y), while the photon may dissociate into a hadronic state $\mathrm{X}, \gamma^{*} p \rightarrow X p^{\prime}(Y)$. The systems are separated by a large rapidity gap. The diffractive exchange object (pomeron) has vacuum quantum numbers and carries away a fraction $x_{\mathbb{P}}$ of the initial proton longitudinal momentum and a momentum transfer $t$ from the incoming to the outgoing proton. It was shown that in quantum chromodynamics (QCD) the cross section for diffractive processes in DIS factorises into universal diffractive parton density functions (DPDFs) of the proton and process-dependent hard scattering cross sections [2]. For highly vir-

*Supported by Centre for particle Physics, project No. LC527. tual photons $\left(Q^{2} \gg 0\right)$ the generic formula reads

$\sigma\left(\gamma^{*} p \rightarrow X p\right)=\sum_{i} f_{i}^{\mathrm{D}}\left(x, Q^{2}, x_{\mathbb{P}}\right) \sigma^{\gamma^{*} i}\left(x, Q^{2}\right) .(1)$

Here, the DPDFs $f_{i}^{\mathrm{D}}\left(x, Q^{2}, x_{\mathbb{P}}\right)$ are integrated over squared four-momentum transferred at the proton vertex $t$ and the sum runs over all contributing partons. Factorisation holds for inclusive and exclusive processes, provided $Q^{2}$ is high enough and if higher twist corrections are neglected. When applied to inclusive diffractive DIS it allows to extract the proton DPDFs from the data. Both the H1 and ZEUS collaborations have performed such fits, assuming Regge factorisation for the DPDFs [3],

$f_{i}^{\mathrm{D}}\left(x, Q^{2}, x_{\mathbb{P}}\right)=f_{\mathbb{P}}\left(x_{\mathbb{P}}\right) f_{i}^{\mathbb{P}}\left(\beta=x / x_{\mathbb{P}}, Q^{2}\right)$

where $\beta$ is fractional momentum of the quark struck by the $\gamma^{*}$ and the pomeron flux $f_{\mathbb{P}}\left(x_{\mathbb{P}}\right)$ is taken from Regge phenomenology. In these fits a small reggeon contribution was taken into account $[4,5]$. It was shown, however, that inclusive diffractive scattering data alone are not able to constrain the diffractive gluon density at high momentum fractions. Measurements of diffractive dijet production in DIS or open charm $\left(D^{*}\right)$ can directly constrain the diffractive gluon density at high momentum fraction. In $[6,7]$ combined Next to Leading Order (NLO) QCD fits have been performed to both inclusive diffractive cross sections and diffractive dijet cross sections in DIS, thus 
giving access to diffractive parton densities in the region of high momentum fractions.

\section{Tests of QCD factorisation}

Using the HERA DPDFs in LO, QCD overestimates diffractive dijet production at the Tevatron by approximately one order of magnitude [8]. This discrepancy was successfully explained in terms of rescattering and absorption: typical models that describe suppression (characterised by a rapidity gap survival probability) assume there are secondary interactions of spectators [911]. In order to investigate the size of this suppression, NLO QCD calculations using factorisation assumptions were confronted with experimental results from HERA. It was observed by both the $\mathrm{H} 1$ and ZEUS collaborations $[12,13]$ that measurements of diffractive $\mathrm{D}^{*}$ meson production are rather well described by NLO QCD calculations based on the HERA DPDFs, without need of suppression. Unfortunately these results suffer from rather large statistical uncertainties.

\section{Dijets in photoproduction}

In LO QCD the photon in photoproduction regime has two components, direct and resolved. In direct photon processes a point-like photon interacts with a parton from the proton, whereas in resolved processes the photon behaves as a source of partons, which subsequently interact with partons from the proton. QCD factorisation is expected to hold in direct diffractive photoproduction. Resolved photon collisions, in contrast, may produce final states which are similar to those in $p \bar{p}$ interactions. They have hadronic remnants which can interact with the proton or with colour singlet exchange spectators. In [11] a suppression factor of size 0.34 is predicted for resolved photon processes. A variable which can be used to separate direct and resolved processes is $x_{\gamma}$, measuring the fraction of the photon momentum participating in the production of the dijet system. The resolved (direct) processes dominate at low (high) $x_{\gamma}$ values.

Diffractive photoproduction (DPHP) of dijets was analysed by both the H1 [14] and ZEUS [15]

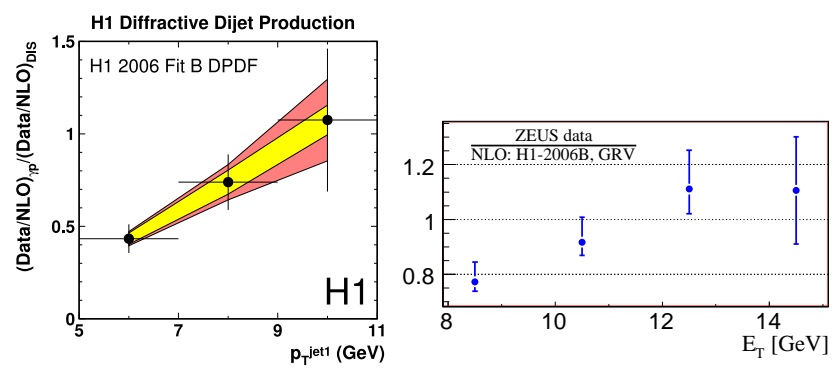

Figure 1. Cross section double ratio of data to NLO prediction for photoproduction and DIS as a function of transverse momentum of the leading jet measured by $\mathrm{H} 1$ (left plot) and cross section ratio of data and NLO for the diffractive photoproduction of dijets vs. $E_{\mathrm{T}}$ of the leading jet as measured by ZEUS (right plot).

collaborations. The $\mathrm{H} 1$ experiment analysed the data with a tagged electron $\left(Q^{2}<0.01 \mathrm{GeV}^{2}\right)$ recorded in the years 1996/97. Diffractive events were selected with a large rapidity gap. The transverse energies for leading and subleading jets were required to be $E_{\mathrm{T} 1}>5 \mathrm{GeV}$ and $E_{\mathrm{T} 2}>4 \mathrm{GeV}$. The ZEUS analysis of dijets in DPHP covered a somewhat different kinematic region, the main difference being higher transverse energies of the leading and subleading jets, $E_{\mathrm{T} 1}>7.5 \mathrm{GeV}$ and $E_{\mathrm{T} 2}>6.5 \mathrm{GeV}$, respectively. In both experiments the jets were identified using the inclusive $k_{\mathrm{T}}$ cluster algorithm in the laboratory frame. The H1 experiment observed a global suppression of the dijet cross sections with respect to NLO QCD calculations by a factor of about 0.5 . The ZEUS data were compatible with no suppression. Neither experiment did observe a difference in suppression for the resolved part $\left(x_{\gamma}<0.75\right)$ as compared to the direct part of the cross section $\left(x_{\gamma}>0.75\right)$, in contradiction to predictions from suppression models. The observed difference in suppression between $\mathrm{H} 1$ and ZEUS was compatible with a dependence of the suppression on $E_{\mathrm{T}}$. The cross section double ratio of data and NLO prediction for DPHP and diffrac- 

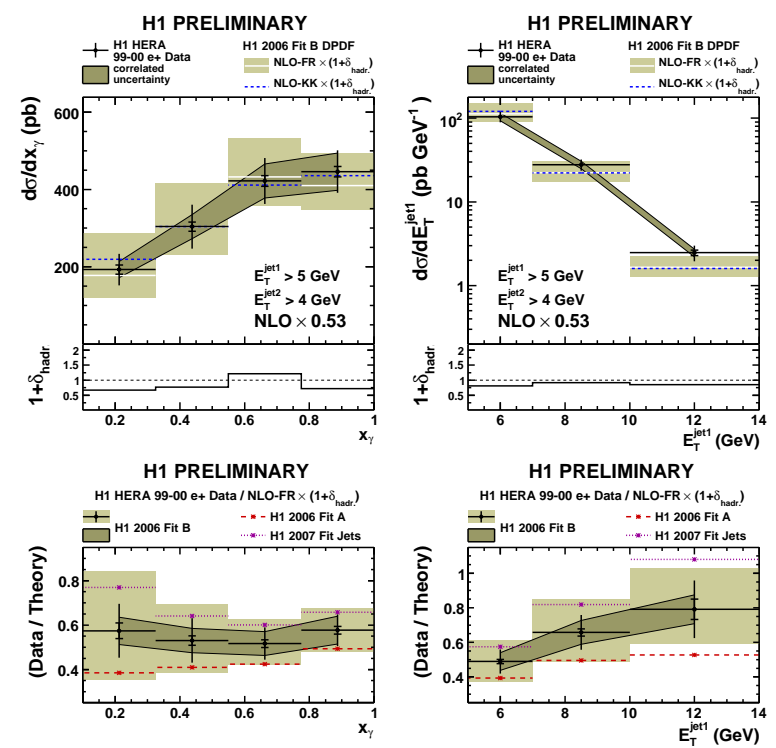

Figure 2. Differential cross section for the diffractive photoproduction of dijets as a function of $x_{\gamma}$ and $E_{\mathrm{T} 1}$ for the lower $E_{\mathrm{T}}$ cut scenario compared to NLO scaled calculations (upper plots). NLO predictions are corrected for hadronisation effects shown bellow the figure. The lower plots show the corresponding ratios of the data to NLO calculated cross sections.

tive DIS measured by $\mathrm{H}^{2}$, as well as the ratio of the ZEUS DPHP data to the NLO predictions [16] are shown as a function of $E_{\mathrm{T}}$ of the leading jet in Figure 1. The data indicate an increase of the suppression factor with increasing $E_{\mathrm{T}}$.

A more detailed study was based on $\mathrm{H} 1$ data of dijets in photoproduction taken in the years $1999 / 2000$ and corresponding to a three times larger data sample. The study was performed using two $E_{\mathrm{T}}$ cut schemes [17]. The first scheme was identical to the older analysis [14] with $E_{\mathrm{T} 1}>$ $5 \mathrm{GeV}$. The second scheme applied cuts close to the ones used by ZEUS [15], $E_{\mathrm{T} 1}>7.5 \mathrm{GeV}$. The results were compared to NLO calculations using three H1 DPDF fits (A, B, Jets) and two independent NLO QCD codes, one by Frixione

\footnotetext{
$\overline{{ }^{2} \text { left plot derived from }}$ [14], thanks to S.Schaetzel
}
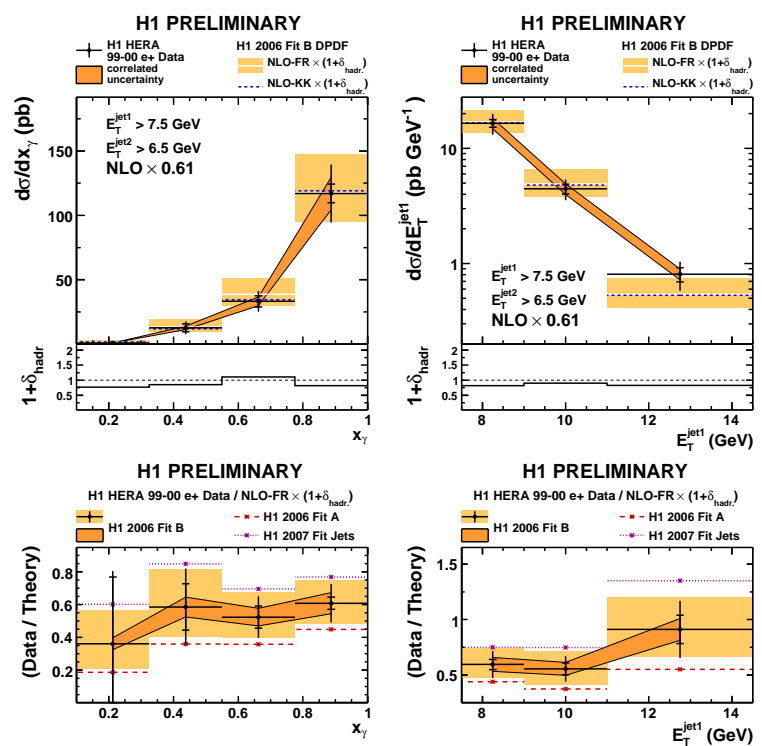

Figure 3. Differential cross section for the diffractive photoproduction of dijets as a function of $x_{\gamma}$ and $E_{\mathrm{T} 1}$ for the higher $E_{\mathrm{T}}$ cut scenario compared to NLO scaled calculations (upper plots). NLO predictions are corrected for hadronisation effects shown bellow the figure. The lower plots show the corresponding ratios of the data to NLO calculated cross sections.

and Ridolfi [18] and the other by Klasen and Kramer [19]. It was verified that both codes give the same results.

In the lower $E_{\mathrm{T}}$ cut scenario the results of the previous H1 analysis were confirmed. The best description of the measured cross sections was obtained by the NLO calculations with fit B and a global suppression of about 0.5 (see Fig. 2). The calculations using DPDF fits A or Jets give similar results. As can be seen in Fig. 3, in the higher $E_{\mathrm{T}}$ cut scenario the best description of the data was obtained with a suppression factor of about 0.6 for fit B. Although the uncertainties are significant, these measurements of the suppression factors seem to support the idea of an $E_{\mathrm{T}}$ dependent suppression in dijet photoproduction.

New DPDF fits ( $\mathrm{C}$ and $\mathrm{S}$, using inclusive and 


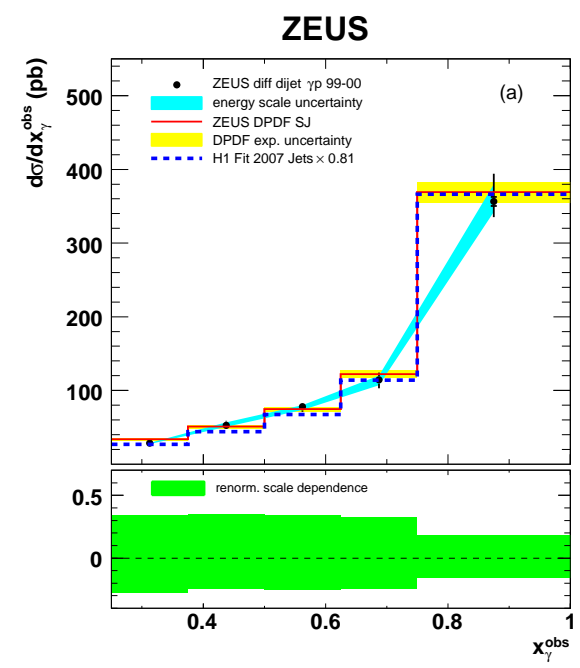

Figure 4. Differential cross section for the diffractive photoproduction of dijets as a function of $x_{\gamma}$ in comparison with DPDFs H1 fit Jets [6] and ZEUS inclusive and dijet fit [20].

dijet DIS data) have been provided by the ZEUS collaboration [20]. The ZEUS measurements of cross sections in diffractive dijet photoproduction [15] were compared to NLO predictions using fit $\mathrm{S}$ and the $\mathrm{H} 1$ fit Jets. No suppression was observed, as shown in Figs. 4 and 5. Note however, that a suppression of 0.8 , as seen in the $\mathrm{H} 1$ high $E_{\mathrm{T}}$ cut scenario (using the fit Jets) is within errors in agreement with the ZEUS result. It is also interesting to note that the hadronisation corrections which are applied to the NLO predictions in the analyses by both experiments are different $[14,17,15]$, correcting NLO predictions in a opposite way and thus making the interpretation of the results even more difficult.

Measurements of ratios of diffractive to inclusive dijet photoproduction cross sections have been proposed [21] as a further test of the dynamics of gap survival issues. Their potential is in the partial cancellations of both experimental and theoretical uncertainties such as photon structure functions or factorisation and renormal-

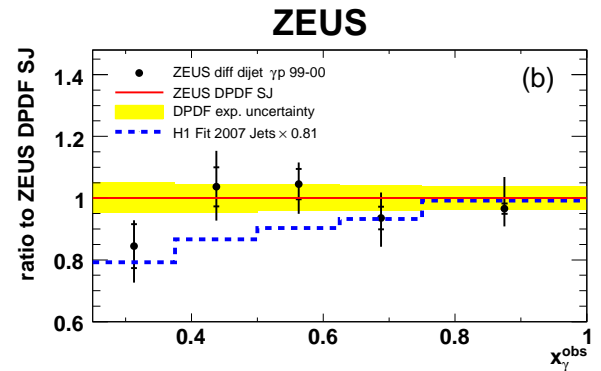

Figure 5. Ratio of the cross section for the diffractive photoproduction of dijets to NLO calculations with different fits as a function of $x_{\gamma}$.

isation scale choices. Such ratios have been measured by $\mathrm{H} 1$ as a function of several variables [22], using identical cuts to select the diffractive and non-diffractive samples, with the exception of the large rapidity gap requirement. The results are shown in Figure 6 . The ratio as a function of $x_{\gamma}$ is believed to be the most sensitive variable to factorisation breaking and gap survival factors $[21,23]$. The data are compared to ratios of RAPGAP and PYTHIA predictions. For inclusive photoproduction, PYTHIA with and without multiple interactions (MI) has been studied, because it is known that for low transverse momenta regime of inclusive photoprodution the underlying event or multi-parton interactions play an important role [24]. The RAPGAP/PYTHIA MI ratio describes data rather well, however the large uncertainties in modelling the underlying event prevent any strong conclusions to be made.

\section{Summary}

The diffractive processes at HERA were analysed in view of a possible breaking of QCD factorisation. The measured cross sections for diffractive dijet photoproduction were compared with NLO QCD predictions based on various sets of parton distribution functions. The dijet photoproduction cross section for jets with low transverse energy $E_{\mathrm{T}}$ of the leading jet, $\left(E_{\mathrm{T} 1}>\right.$ $5 \mathrm{GeV})$, are overestimated by NLO QCD predic- 

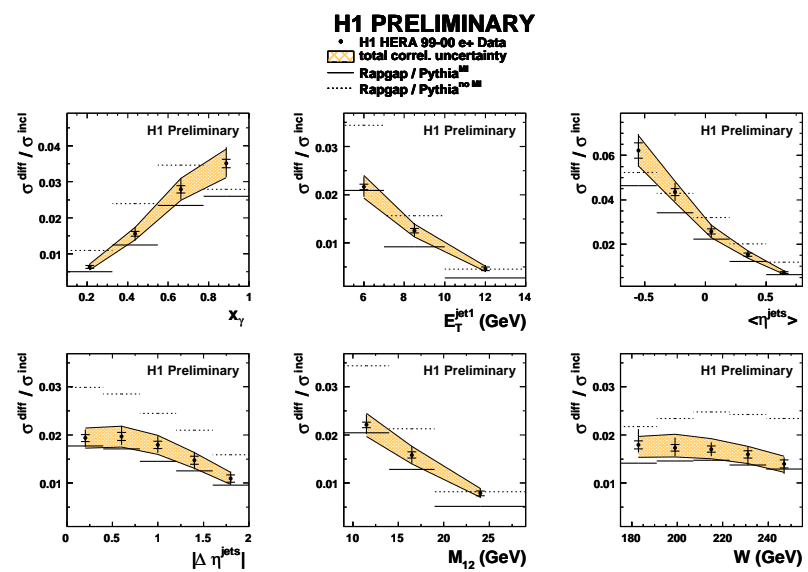

Figure 6. Observed ratios of diffractive to inclusive cross sections compared to predictions obtained with RAPGAP and PYTHIA. The predicted ratios are shown with multiple interactions (MI) in PYTHIA switched on or off. The points show the data ratio, the inner error bars are statistical and outer error bars show statistical and uncorrelated uncertainties added in quadrature. The correlated uncertainty is indicated with hatched band.

tions. Hints for a dependence of the suppression factor on the $E_{\mathrm{T}}$ of the leading jet were found. The ZEUS collaboration did not observe suppression of data in the sample with $E_{\mathrm{T}}$ of the leading jet, $E_{\mathrm{T} 1}>7.5 \mathrm{GeV}$. Within uncertainties this is not in contradiction to a suppression of $0.8 \mathrm{ob}-$ served by $\mathrm{H} 1$ in a similar analysis. The ratio of diffractive to inclusive dijet differential cross sections were measured as a function of several variables. However, large uncertainties in modelling the underlying event for non-diffractive dijet production prevent any strong conclusions to be made.

\section{REFERENCES}

1. ZEUS Coll., M.Derrick et al., Phys.Lett. B 316 (1993) 207

2. J.Collins, Phys. Rev. D 57 (1998) 3051.
3. G.Ingelman, P.E.Schlein, Phys. Lett. B152, 256 (1985).

4. H1 Coll., A.Aktas et al., Eur.Phys. J. C48, 715 (2006).

5. ZEUS Coll.,S.Chekanov et al., Eur. Phys. J. C38, 43, (2004).

6. H1 Coll., A.Aktas et al., JHEP 0710 (2007) 042

7. ZEUS Coll., S.Chekanov et al., Eur. Phys. J.C52 (2007) 813.

8. CDF Coll., T.Affolder et al., Phys. Rev. Lett. 84 (2000) 5043.

9. Y.Dokshitzer et al., Phys. Lett. B 274 (1992),116.

10. J.Bjorken, Phys. Rev. D 47 (1993) 101.

11. A.Kaidalov et al., Eur. Phys. J. C 21 (2001) 521.

12. H1 Coll., A.Aktas et al., Eur. Phys. J. C50, (2007) 1 .

13. ZEUS Coll., S. Chekanov et al., Nucl. Phys. B672 (2003), 3.

ZEUS Coll., S. Chekanov et al., Eur. Phys. J. C51 (2007) 301.

14. H1 Coll., A.Aktas et al., Eur. Phys. J. C51 (2007) 549.

15. ZEUS Coll., S.Chekanov et al., Eur.Phys. J. C55 (2008) 177.

16. W.Slominski, Dijets in Diffractive DIS and Photoproduction, Proceedings of DIS08 (London), April 2008.

17. K.Cerny, Diffractive Photoproduction of Dijets in ep Collisions at HERA, Proceedings of DIS08 (London), April 2008.

18. S. Frixione et al., Nucl. Phys.B467 (1996) 399.

19. M.Klasen, G.Kramer, Eur. Phys. J. C38 (2004) 93.

20. ZEUS Coll., W. Slominski, Factorization Tests, Proceedings of DIS09 (Madrid), April 2009.

21. A. Kaidalov, V.Khoze et al., Phys. Lett. B567 (2003), 61.

22. P. Newman, Diffractive Dijets in Photoproduction, Proceedings of DIS09 (Madrid), April 2009.

23. M.Klasen, G.Kramer, Eur. Phys. J. C38 (2004) 93.

24. H1 Coll., C.Adloff et al., Eur. Phys. J. C29 (2003) 497. 